\title{
A PROOF OF THE 4-VARIABLE CATALAN POLYNOMIAL OF THE DELTA CONJECTURE
}

\author{
MIKE ZABROCKI
}

\begin{abstract}
In The Delta Conjecture HRW15, Haglund, Remmel and Wilson introduced a four variable $q, t, z, w$-Catalan polynomial, so named because the specialization of this polynomial at the values $(q, t, z, w)=(1,1,0,0)$ is equal to the Catalan number $\frac{1}{n+1}\left(\begin{array}{c}2 n \\ n\end{array}\right)$. We prove the compositional version of this conjecture (which implies the noncompositional version) that states that the coefficient of $s_{r, 1^{n-r}}$ in the expression $\Delta_{h_{\ell}} \nabla C_{\alpha}$ is equal to a weighted sum over decorated Dyck paths.
\end{abstract}

This paper is dedicated to the memory of Jeffery Remmel (1948-2017).

\section{INTRODUCTION}

In the search for a representation theoretical interpretation for Macdonald symmetric functions, Haiman defined the module of diagonal harmonics [Hai94] as a quotient of the polynomial ring in two sets of $n$ variables. For a given integer $n$, the diagonal harmonics are a bi-graded $S_{n}$-module with dimension $(n+1)^{n-1}$. Garsia and Haiman GarHai96] took a (at the time conjectured) formula for the bi-graded Frobenius characteristic of the diagonal harmonics and defined for each $n$ a rational function in two parameters $q$ and $t$ which is equal to the bi-graded multiplicity of the alternating representation in the module. This expression is known as the $q, t$-Catalan polynomial [GarHai96] since at $q=t=1$ it specializes to the Catalan number $\frac{1}{n+1}\left(\begin{array}{c}2 n \\ n\end{array}\right)$. In 2000, Garsia and Haglund Hag03, GarHag02 announced a proof that the $q, t$-Catalan was a polynomial in $q$ and $t$ with non-negative integer coefficients and provided a combinatorial interpretation for the expression in terms of Dyck paths.

Important progress was made in the development of that proof through the introduction of the notation of two linear symmetric function operators $\nabla$ and $\Delta_{f}$ that have Macdonald symmetric functions as eigen-functions [BG99, BGHT99. The expression $\nabla\left(e_{n}\right)$ was conjectured to be equal to the Frobenius image of the character of the module of diagonal harmonics and the $q, t$-Catalan polynomial is the coefficient of $e_{n}$ in this expression. The operators $\nabla$ and $\Delta_{f}$ gave notation to extend the types of symmetric function expressions which were conjectured to be Schur positive for representation theoretic reasons to expressions which are conjectured to be Schur positive because of computer experimentation.

Haglund conjectured Hag03 and shortly after Garsia and Haglund GarHag02 proved a combinatorial interpretation for the $q, t$-Catalan polynomial. They showed that there were two statistics on Dyck paths (called area and bounce) such that the rational expression for 
the $q, t$-Catalan is equal to the sum over all Dyck paths $D$ with weight $q^{\text {area }(D)} t^{\text {bounce }(D)}$. Around this same period, Haiman [Hai02] proved the conjecture that $\nabla\left(e_{n}\right)$ was equal to the Frobenius image of the graded character of the diagonal harmonics. Haiman also guessed at a second statistic (dinv, short for diagonal inversions) such that the $q, t$-Catalan is equal to the sum over all Dyck paths with weight $q^{\operatorname{dinv}(D)} t^{\operatorname{area}(D)}$ and Haglund later showed with a bijection why the two combinatorial expressions are equivalent.

With the conjectures on the q,t-Catalan polynomial resolved, Haglund, Haiman, Loehr, Remmel and Ulyanov [HHLRU05] extended the combinatorial interpretations for the coefficient of $e_{n}$ in $\nabla\left(e_{n}\right)$ to other coefficients. They conjectured the coefficient of any monomial symmetric function in terms of labelled Dyck paths (also known as parking functions) and this became known as the Shuffle Conjecture. The Shuffle Conjecture takes its name because the coefficient of a monomial is equal to the number of labelled Dyck paths whose reading word is a shuffle of segments of length the parts of the partition.

Researchers also considered coefficients of $\nabla$ and $\Delta_{f}$ acting on other symmetric functions and extended the combinatorial interpretations to coefficients in these expressions (e.g. [EHKK03, Hag04, CL06, LW07, LW08, and for a survey of results in this area up to 2008 see Hag08).

In particular, a refinement of the Shuffle Conjecture was proposed by Haglund, Morse and the author [HMZ12] that gave a symmetric function expression for the labelled Dyck paths which touch the diagonal at a given composition. Some progress on this Compositional Shuffle Conjecture was made [GXZ10, Hic10, DGZ13, Hic14, GXZ14a, GXZ14b] before it was finally proven by Carlsson and Mellit CM15. By the time that Carlsson and Mellit had announced their proof, there was already a rational slope version of the compositional shuffle conjecture BGLX16]. The arms race of conjecture vs. proof in this area did not stay out of balance for long and a proof of this result was announced in 2016 by Mellit [Mel16].

Haglund, Remmel and Wilson HRW15] recently announced a conjecture for some combinatorial expressions involving $\Delta_{f}$ and $\nabla$ in a sequence of conjectures that generalize the Shuffle Conjecture from labelled Dyck paths to decorated labelled Dyck paths and called this the Delta Conjecture. There does not currently exist a compositional version of this conjecture which might be helpful if progress is to be made on proving it.

They noticed however that the coefficients of a Schur function indexed by a hook in the expression $\Delta_{h_{\ell}} \nabla e_{n}$ had similar behavior to the $q, t$-Catalan GarHai96, GarHag02 and $q, t$ Schröder EHKK03, Hag04 and they proposed a four parameter expression $C_{n}(q, t, w, z)$ and a combinatorial interpretation for this expression in terms of decorated Dyck paths. In fact they proposed two combinatorial interpretations and one of them is compatible with the compositional refinement proposed by Haglund, Morse and the author [HMZ12]. It is this conjecture that we shall prove here.

Although this is not precisely how the combinatorial interpretation was formulated in [HRW15, we will present it here in terms of decorated Schröder paths. Schröder paths were used as a combinatorial description for the coefficients of Schur function indexed by a hook in the expression $\nabla\left(e_{n}\right)$ in [EHKK03, Hag04]. In this paper we will give a combinatorial 
description for the coefficient of a Schur function indexed by a hook in the expression $\Delta_{h_{\ell}} \nabla\left(e_{n-\ell}\right)$ as Schröder paths with $\ell$ vertical segments decorated with a $\circ$ symbol.

In fact, a Schröder path is simply a Dyck path with some of the peaks in the Dyck path changed to $N E$-diagonal steps. In all of the Schröder paths we will also insist that the right most peak in the highest diagonal not have a $N E$-diagonal step 11

A Schröder path is a generalization of a Dyck path that is a lattice path in the $n \times n$ square that start in the South-West corner and go to the North-East corner allowing for steps North, East and diagonal steps which are North-East such that the path stays above the South-West/North-East diagonal. A o-decorated Schröder path is a Schröder path in which some of the vertical steps which are not peaks are decorated with a $\circ$. We will show that the coefficient of $s_{k+1,1^{n-k-\ell-1}}$ in $\Delta_{h_{\ell}} \nabla e_{n-\ell}$ is a $q, t$ enumeration of the o-decorated Schröder paths of length $n$ with $k$ diagonal North-East steps and $\ell$ vertical segments decorated with $\circ$. In particular we will show that $\left\langle\Delta_{h_{\ell}} \nabla\left(e_{n-\ell}\right), e_{n-\ell}\right\rangle$ is a positive polynomial in $q, t$ that enumerates o-decorated Dyck paths.

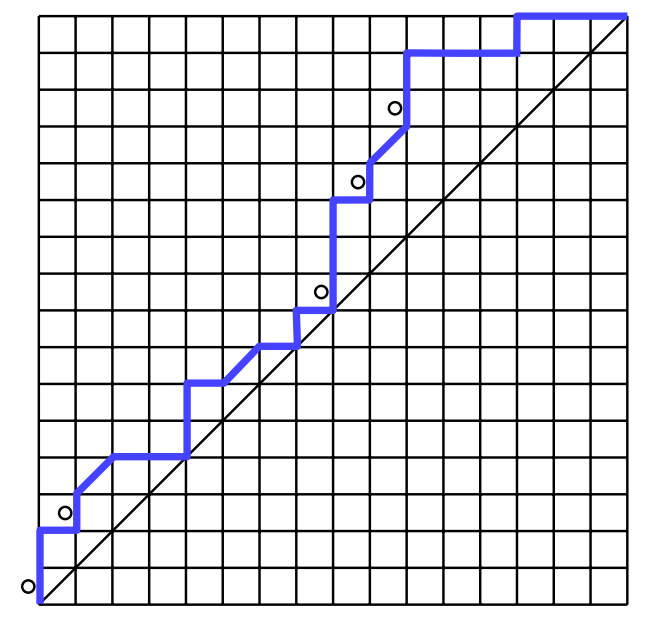

Figure 1. An example of a o-decorated Schröder path. In this example, $\operatorname{rise}_{\circ}(D)=5$ because there are five o-decorated vertical segments and $\operatorname{diag}(D)=3$ because there are three NE-diagonal steps. The usual touch composition is $(4,3,1,8)$, but the rise-touch composition is equal to $(2,3,1,5)$ because two vertical segments are o-decorated in the first touch segment and three vertical segments are o-decorated in the last touch segment.

\footnotetext{
${ }^{1}$ In an early version of HRW15, the combinatorial interpretation was stated in terms of o-decorated Dyck paths where there is a difference in the decorations on the peaks and double rises. The latest version does not express the combinatorial interpretation for the coefficients in terms of decorated Dyck paths at all, but it is a useful construction in relating the right and left hand side of Theorem 11. Here we use Schröder paths to distinguish the o-decorations on the peaks (which are diagonal edges here) from those on the double rises.
} 
For a given o-decorated Schröder path $P$, the number of o-decorations on the path will be denoted $\operatorname{rise}_{\circ}(P)$ and the number of diagonal NE steps will be denoted $\operatorname{diag}(P)$. The positions where the Schröder path touches the diagonal divides the path into segments and determines a composition, $\operatorname{touch}(P)=\left(\alpha_{1}, \alpha_{2}, \ldots, \alpha_{\ell(\alpha)}\right)$ where $\alpha_{i}$ is the length of the $i^{t h}$ segment. We will also consider the rise-touch composition touch ${ }_{\circ}(P)=\left(\beta_{1}, \beta_{2}, \ldots, \beta_{\ell(\alpha)}\right)$ where $\beta_{i}$ is equal to $\alpha_{i}$ minus the number of o-decorations in the $i^{\text {th }}$ segment. There are two additional statistics on these paths $\operatorname{area}_{\circ}(P)$ and $\operatorname{dinv}_{\circ}(P)$ which we will explain in detail in Section 3 ,

Namely we will show the following theorem (this is Theorem 11; note: we leave the definition of the statistics dinv。 and area。 to Section 3):

Theorem 1. For non-negative integers $n, k, \ell$ and a composition $\alpha$ of size $n-\ell$,

$$
\left\langle\Delta_{h_{\ell}} \nabla C_{\alpha}, s_{k+1,1|\alpha|-k-1}\right\rangle=\sum_{\substack{P \in \mathbf{S c h}_{n}^{\circ} \\ \operatorname{diag}(P)=k, \text { rise }_{\circ}(P)=\ell \\ \operatorname{touch}_{\circ}(P)=\alpha}} q^{\operatorname{dinv}_{\circ}(P)} t^{\text {area }_{\circ}(P)}
$$

where the sum is over all o-decorated Schröder paths with $k$ NE-diagonal steps and $\ell$ odecorated rises and rise-touch composition equal to $\alpha$.

The symmetric function $C_{\alpha}$ which appears in this theorem is compositional form of a Hall-Littlewood symmetric function which was introduced in HMZ12. The definition appears in section 2.4. By Proposition 5.2 of [HMZ12], $e_{n}=\sum_{\alpha=n} C_{\alpha}$ and hence we have given a combinatorial interpretation for $\left\langle\Delta_{h_{\ell}} \nabla e_{n-\ell}, s_{k+1,1^{n-\ell-k-1}}\right\rangle$.

Note that the resolution of this conjecture does not prove all of the conjectures made in Section 7 of [HRW15] because there was a second combinatorial interpretation stated for the coefficient $\left\langle\Delta_{h_{\ell}} \nabla e_{n-\ell}, s_{k+1,1^{n-k-1}}\right\rangle$ that does not seem to be compatible with the compositional version and our techniques depend strongly on compatibility with the compositional construction.

\section{Symmetric FunCtions}

The results related to Macdonald symmetric functions that we will use here almost all come from a series of early papers on the subject Mac88, G92, GarHai95, GarHai96, BG99, BGHT99, GHT99, GarHag02. These results have proven to be very prescient in the utility of the identities, notation and techniques developed. We will be able to prove our symmetric function recurrence by using the groundwork paved in these references. The book by J. Haglund Hag08 collects many of the identities that we will use in a review of the literature and hence will provide a useful reference for their use. The only additional ingredient that we will use are the creation operators and symmetric functions introduced in HMZ12 which play an important role in developing recurrences for the coefficients in which we are interested.

2.1. Symmetric function notation. The main reference we will use for symmetric functions is Mac95]. The standard bases of the symmetric functions that will appear in our calculations the complete $\left\{h_{\lambda}\right\}_{\lambda}$, elementary $\left\{e_{\lambda}\right\}_{\lambda}$, power $\left\{p_{\lambda}\right\}_{\lambda}$ and Schur $\left\{s_{\lambda}\right\}_{\lambda}$ bases. 
The ring of symmetric functions can be thought of as the polynomial ring in the power sum generators $p_{1}, p_{2}, p_{3}, \ldots$. As we are working with Macdonald symmetric functions involving two parameters $q$ and $t$ we will consider this polynomial ring over the field $\mathbb{Q}(q, t)$.

We will make extensive use of plethystic notation in our calculations and arbitrary alphabets. This is a notational addition that introduces union and difference of alphabets. Alphabets will be represented as sums of monomials $X=x_{1}+x_{2}+x_{3} \ldots$ and then the expression $f[X]$ represents the symmetric function $f$ as an element of $\Lambda$ with $p_{k}$ replaced by $x_{1}^{k}+x_{2}^{k}+x_{3}^{k}+\cdots$. We have the identities that $p_{k}[X+Y]=p_{k}[X]+p_{k}[Y], p_{k}[X-Y]=$ $p_{k}[X]-p_{k}[Y]$, and on the elementary and homogeneous bases we also have the alphabet addition formulae which say

$$
e_{n}[X+Y]=\sum_{k=0}^{n} e_{k}[X] e_{n-k}[Y] \text { and } h_{n}[X+Y]=\sum_{k=0}^{n} h_{k}[X] h_{n-k}[Y] .
$$

The notation $\epsilon$ is a common tool to express a second sort of negative sign when working with symmetric functions with alphabets where $p_{k}[\epsilon X]=(-1)^{k} p_{k}[X]$. This is different from the negative of the alphabet expressed as $p_{k}[-X]=-p_{k}[X]$. In general $f[-\epsilon X]=(\omega f)[X]$ where $\omega$ is the fundamental algebraic involution which sends $e_{k}$ to $h_{k}, s_{\lambda}$ to $s_{\lambda^{\prime}}$ and $p_{k}$ to $(-1)^{k-1} p_{k}$.

There is a special element $\Omega$ in the completion of the symmetric functions that we will be using. It is defined as $\Omega=\sum_{n>0} h_{n}$. It has the property for arbitrary alphabets $X$ and $Y, \Omega[X+Y]=\Omega[X] \Omega[Y]$. In addition, it has the property that for any two dual basis $\left\{a_{\lambda}\right\}_{\lambda}$ and $\left\{b_{\lambda}\right\}_{\lambda}$ with respect to the standard scalar product $\left\langle s_{\lambda}, s_{\mu}\right\rangle=\chi(\lambda=\mu)$, we have

$$
\Omega[X Y]=\sum_{\lambda} a_{\lambda}[X] b_{\lambda}[Y]
$$

2.2. Macdonald symmetric function toolkit and $q, t$ notation. Macdonald symmetric functions that are used here are a transformation of the bases presented in Mac95. They are the symmetric functions that are the Frobenius image of the Garsia-Haiman modules GarHai93 indexed by a partition. The symmetric functions

$$
\tilde{H}_{\mu}[X ; q, t]=\sum_{\lambda \vdash|\mu|} K_{\lambda \mu}(q, 1 / t) t^{n(\mu)} s_{\lambda}[X]
$$

where $K_{\lambda \mu}(q, t)$ are the Macdonald $q, t$-Kostka coefficients and $n(\mu)=\sum_{i \geq 1}(i-1) \mu_{i}$. The basis elements $\left\{\tilde{H}_{\mu}\right\}_{\mu}$ are orthogonal with respect to the scalar product

$$
\left\langle p_{\lambda}, p_{\mu}\right\rangle_{*}=\chi(\lambda=\mu)(-1)^{|\lambda|+\ell(\lambda)} \prod_{i=1}^{\ell(\lambda)}\left(1-q^{\lambda_{i}}\right)\left(1-t^{\lambda_{i}}\right)
$$

and are sometimes defined by this property.

If we identify the partition $\mu$ with the collection of cells $\left\{(i, j): 1 \leq i \leq \mu_{i}, 1 \leq j \leq \ell(\mu)\right\}$, then for each cell $c \in \mu$ we refer to the the arm, leg, co-arm and co-leg (denoted respectively 


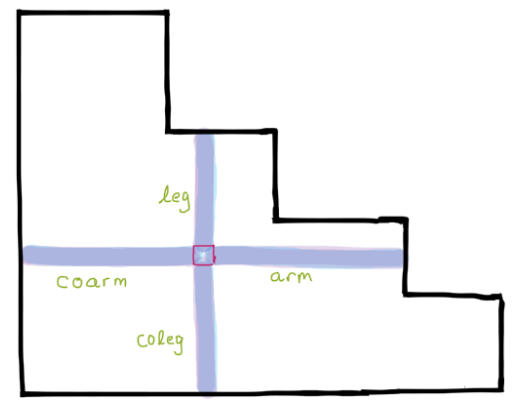

FIGURE 2. arm, leg, co-arm and co-leg of a cell of the diagram

as $\left.a_{\mu}(c), \ell_{\mu}(c), a_{\mu}^{\prime}(c), \ell_{\mu}^{\prime}(c)\right)$ as the number of cells in the segments labeled in Figure 2. The typical shorthand for the polynomial expressions in $q$ and $t$ are

$B_{\mu}=\sum_{c \in \mu} q^{a_{\mu}^{\prime}(c)} t^{\ell_{\mu}^{\prime}(c)}, T_{\mu}=\prod_{c \in \mu} q^{a_{\mu}^{\prime}(c)} t^{\ell_{\mu}^{\prime}(c)}$ and $w_{\mu}=\prod_{c \in \mu}\left(q^{a_{\mu}(c)}-t^{\ell_{\mu}(c)+1}\right)\left(t^{\ell_{\mu}(c)}-q^{a_{\mu}(c)+1}\right)$.

Also set $M=(1-q)(1-t)$ and $D_{\mu}=M B_{\mu}-1$.

The following linear operators were introduced in BG99, BGHT99] which are at the basis of the conjectures relating symmetric function coefficients and $q, t$-combinatorics in this area. Define

$$
\nabla\left(\tilde{H}_{\mu}\right)=T_{\mu} \tilde{H}_{\mu} \text { and } \Delta_{f}\left(\tilde{H}_{\mu}\right)=f\left[B_{\mu}\right] \tilde{H}_{\mu} .
$$

Note that if $n=|\mu|$, then $e_{n}\left[B_{\mu}\right]=T_{\mu}$, hence for a symmetric function $f$ of homogeneous degree $n, \Delta_{e_{n}}(f)=\nabla(f)$, so the operators $\Delta_{f}$ are seen as a more general operator than $\nabla$.

Following other references and introduce the shorthand notation $f^{*}[X]=f\left[\frac{X}{M}\right]$. This notation can then be used to relate the $*$-scalar product with the usual scalar product $\langle f, g\rangle$ where the Schur functions are orthonormal since $\langle f, g\rangle=\left\langle f, \omega g^{*}\right\rangle_{*}$. It is known that $\left\langle\tilde{H}_{\lambda}, \tilde{H}_{\mu}\right\rangle_{*}=\chi(\lambda=\mu) w_{\lambda}$, then follows that

$$
\Omega\left[\frac{-\epsilon X Y}{M}\right]=\sum_{n \geq 0} e_{n}^{*}[X Y]=\sum_{\mu \vdash n} \frac{\tilde{H}_{\mu}[X] \tilde{H}_{\mu}[Y]}{w_{\mu}} .
$$

We will use one of the forms of Macdonald-Koornwinder reciprocity in our calculations (see [Mac95] p. 332 or [GHT99]),

$$
\frac{\tilde{H}_{\mu}\left[1+u D_{\lambda}\right]}{\prod_{c \in \mu}\left(1-u t^{\ell^{\prime}(c)} q^{a^{\prime}(c)}\right)}=\frac{\tilde{H}_{\lambda}\left[1+u D_{\mu}\right]}{\prod_{c \in \lambda}\left(1-u t^{\ell^{\prime}(c)} q^{a^{\prime}(c)}\right)} .
$$

The form of this identity that we are most interested here is found by setting $u=1 / u$, clearing the denominators, and letting $u \rightarrow 0$ to obtain

$$
\tilde{H}_{\mu}\left[D_{\nu}\right]=(-1)^{|\mu|+|\nu|} \tilde{H}_{\nu}\left[D_{\mu}\right] \frac{T_{\mu}}{T_{\nu}} .
$$


2.3. Pieri rules and summation formulae. Define coefficients $h_{1}^{\perp} \tilde{H}_{\mu}=\sum_{\nu \rightarrow \mu} c_{\mu \nu} \tilde{H}_{\nu}$ and $h_{1} \tilde{H}_{\nu}=\sum_{\mu \leftarrow \nu} d_{\mu \nu} \tilde{H}_{\mu}$. It was proven in GarHai95. (Corollary 1.1) that they are related by the identity,

$$
d_{\gamma \tau}=M c_{\gamma \tau} \frac{w_{\tau}}{w_{\gamma}}
$$

The following identity has been used frequently in work on the Shuffle Conjecture but a full proof did not appear until recently in GHXZ16. For $s \geq 0$,

$$
e_{s-1}\left[D_{\gamma}\right]=(-1)^{s-1} \sum_{\nu \leftarrow \gamma} d_{\nu \gamma}\left(\frac{T_{\nu}}{T_{\gamma}}\right)^{s}+\chi(s=0)
$$

where we have denoted $\chi($ true $)=1$ and $\chi($ false $)=0$ so that the term $\chi(s=0)$ only appears in the case that $s=0$. The other sum of Pieri coefficients for Macdonald polynomials was proven in a $q, t$ hook walk by Garsia and Haiman GarHai95 for $s \geq 0$,

$$
h_{s+1}\left[D_{\gamma}\right]=M t^{s} q^{s} \sum_{\tau \rightarrow \gamma} c_{\gamma \tau}\left(\frac{T_{\gamma}}{T_{\tau}}\right)^{s}-\chi(s=0) .
$$

In order to prove the combinatorial formula for the $q, t$-Catalan polynomial, Garsia and Haglund introduced a generalization of the Pieri coefficients and proved a summation formula which we will use here. They defined coefficients $d_{\mu \nu}^{f}$ and $c_{\mu \nu}^{f \perp}$ where $\nu \subseteq \mu$ as

$$
f \tilde{H}_{\nu}=\sum_{\mu} d_{\mu \nu}^{f} \tilde{H}_{\mu} \quad \text { and } \quad f^{\perp} \tilde{H}_{\mu}=\sum_{\nu} c_{\mu \nu}^{f \perp} \tilde{H}_{\nu}
$$

These coefficients are related by

$$
c_{\mu \nu}^{f \perp} w_{\nu}=d_{\mu \nu}^{\omega f^{*}} w_{\mu} .
$$

The summation formula from GarHag02 (see pp. 698-701) we will use here is

$$
\sum_{\substack{\nu \subseteq \mu \\ m-d \leq|\nu| \leq m}} c_{\mu \nu}^{g \perp}=\left.\nabla^{-1}\left((\omega g)\left[\frac{X-\epsilon}{M}\right]\right)\right|_{X \rightarrow D_{\mu}}
$$

where $\mu \vdash m$ and $g$ is a symmetric function of degree less than or equal to $d$.

2.4. Symmetric functions indexed by compositions and creation operators. The work of Haglund, Morse and the author [HMZ12] extended the Shuffle Conjecture to a compositional refinement. The Compositional Shuffle Conjecture implies the original Shuffle Conjecture, and it was this version of the conjecture that was proven in CM15.

The compositional refinement came by defining for each composition $\alpha$ symmetric functions $B_{\alpha}[X ; q]$ and $C_{\alpha}[X ; q]$. These symmetric functions have the property that the combinatorial expression in terms of labeled Dyck paths for $\nabla B_{\alpha}[X ; q]$ is in terms of paths which touch in at least in the positions specified by the composition $\alpha$ and $\nabla C_{\alpha}[X ; q]$ which touches the diagonal in exactly the positions specified by the composition $\alpha$. 
Both of these symmetric functions are defined in terms of creation operators. For any symmetric function $P[X]$ define

$$
\mathbb{B}_{m} P[X]=\left.P\left[X+\epsilon \frac{(1-q)}{u}\right] \Omega[-\epsilon u X]\right|_{u^{m}}
$$

and

$$
\mathbb{C}_{m} P[X]=\left.(-q) P\left[X+\epsilon \frac{(1-q)}{u}\right] \Omega\left[\frac{\epsilon u X}{q}\right]\right|_{u^{m}} .
$$

Then for any composition $\alpha=\left(\alpha_{1}, \alpha_{2}, \ldots, \alpha_{\ell(\alpha)}\right)$, set $C_{\alpha}=C_{\alpha}[X ; q]=\mathbb{C}_{\alpha_{1}} \mathbb{C}_{\alpha_{2}} \ldots \mathbb{C}_{\alpha_{\ell(\alpha)}}(1)$. We can define the symmetric functions $B_{\alpha}$ in a similar manner, but for our purposes, we only need ([HMZ12] equation (5.11) and (5.12)) the $C_{\alpha}$ symmetric functions and the fact that for $m \geq 0$,

$$
\mathbb{B}_{m}\left(C_{\alpha}\right)=q^{\ell(\alpha)} \sum_{\beta \models m} C_{\alpha, \beta} .
$$

We will denote $\mathbb{B}_{m}^{*}$ and $\mathbb{C}_{m}^{*}$ operators which are dual to $\mathbb{B}_{m}$ and $\mathbb{C}_{m}$ with respect to the *-scalar product (that is $\left\langle\mathbb{B}_{m} f, g\right\rangle_{*}=\left\langle f, \mathbb{B}_{m}^{*} g\right\rangle_{*}$ ). Since $\left\langle e_{n}^{*}[X Y], f[X]\right\rangle_{*}=f[Y]$, then for any symmetric function $f[X]$,

$$
\left\langle\mathbb{B}_{m}^{X} e_{n}^{*}[X Y], f[Y]\right\rangle_{*}=\mathbb{B}_{m} f[X]=\left\langle e_{n}^{*}[X Y], \mathbb{B}_{m}^{Y} f[Y]\right\rangle_{*}=\left\langle\mathbb{B}_{m}^{* Y} e_{n}^{*}[X Y], f[Y]\right\rangle_{*}
$$

where we have denoted $\mathbb{B}_{m}^{X}$ to be the $\mathbb{B}_{m}$ operator acting on the $X$ variables and $\mathbb{B}_{m}^{* Y}$ to be the $*$-dual operator acting on the $Y$ variables. We conclude that the following two expressions be verified by calculating that $\mathbb{B}_{m}^{X} e_{n}^{*}[X Y]=\mathbb{B}_{m}^{* Y} e_{n}^{*}[X Y]$ (and similarly $\left.\mathbb{C}_{m}^{X} e_{n}^{*}[X Y]=\mathbb{C}_{m}^{* Y} e_{n}^{*}[X Y]\right)$. These operators are expressed by the formulae,

$$
\mathbb{B}_{m}^{*} P[X]=\left.P\left[X+\frac{M}{u}\right] \Omega\left[\frac{-u X}{1-t}\right]\right|_{u^{-m}}
$$

and

$$
\mathbb{C}_{m}^{*} P[X]=\left.(-q) P\left[X-\frac{M}{q u}\right] \Omega\left[\frac{-u X}{1-t}\right]\right|_{u^{-m}}
$$

\section{The COMBinAtorial RECURRENCE}

In [HRW15] there are two combinatorial interpretations stated in Conjecture 7.1 for the symmetric function expression $\left\langle\Delta_{h_{\ell}} \nabla e_{n-\ell}, s_{k+1,1^{n-\ell-k-1}}\right\rangle$ and only one of these two seems to be compatible with the coefficient in the expression $\left\langle\Delta_{h_{\ell}} \nabla C_{\alpha}, s_{k+1,1^{n-\ell-k-1}}\right\rangle$ (where $\alpha$ is a composition and $k, \ell \geq 0)$ in terms of decorated Dyck paths.

What we will do in the beginning of this section is to introduce the definitions necessary to state the combinatorial interpretation. In Section 3.1 we state and prove a recurrence on the generating function for the combinatorial objects. Then in Section 4 we will show a symmetric function identity that demonstrates the coefficients also satisfy the same recurrence. This will imply by an inductive argument (because for small values of the indices 
we can verify that the combinatorial values agree with the symmetric function coefficients) that the symmetric function coefficients agree with the combinatorial generating function.

The basic element of the recursive construction given to us by the symmetric function recurrence is a rotation of the first part around to the end of the Dyck path while deleting the first up step and the first right step that touches the diagonal. This combinatorial recurrence and the effect on the area and dinv statistics first appears in [Hic10]. Assuming that we know the size of the piece that is being rotated around, the process is reversible. This is exactly the same sort of recursive construction that appeared in the proofs of certain coefficients of the Compositional Shuffle Conjecture [GXZ10, GXZ14a, GXZ14b].
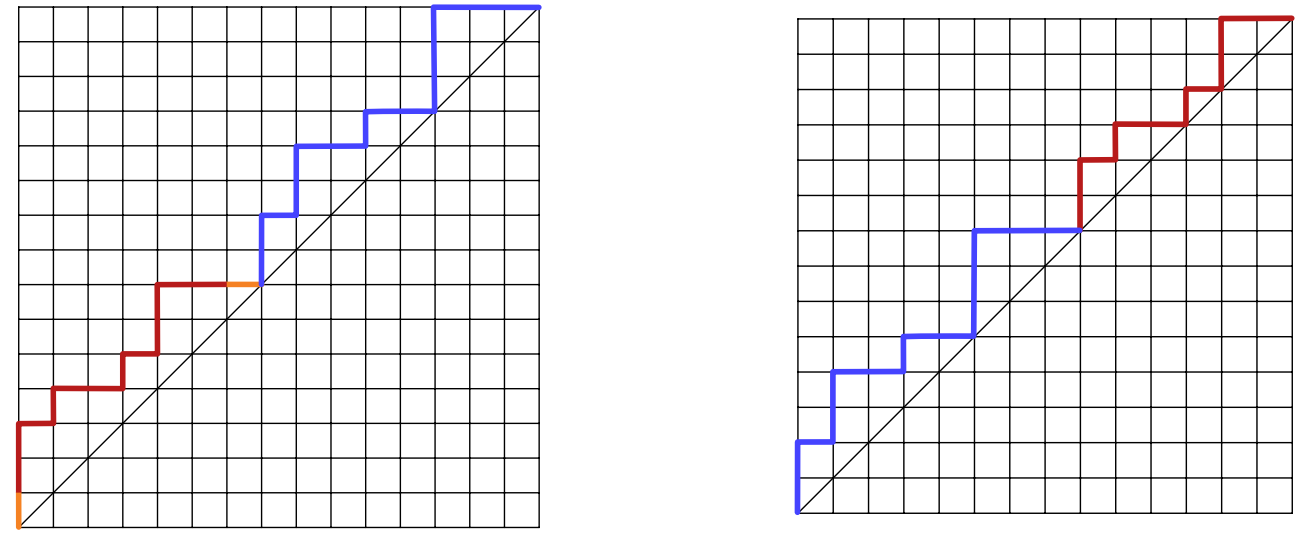

Figure 3. Example of cyclic rotation

The Dyck path on the left first touches the diagonal after 7 vertical/horizontal steps and has area $(D)=17$. The Dyck path on the right has the red part of the path moved to the end with the orange segments removed. The resulting path has area $11=17-(7-1)$.

Vertical edges in a Dyck path come in two types, a peak is a vertical edge followed by a horizontal edge, a double rise is a a vertical edge followed by a second vertical edge. A Schröder path is a Dyck path with some of the peaks changed to $N E$-diagonal edges. The Schröder paths we will work with will have the restriction that the rightmost peak in the highest diagonal cannot be a $N E$-diagonal edge. A decorated Schröder path will be a Schröder path with some of the vertical edges which are not peaks decorated with a o. Since a o-decorated Schröder path is just a Dyck path with some of the vertical edges either decorated or diagonal, we will explain the recurrence on the Dyck path and assume that the decorations travel with the edges in the recurrence. In this way we will identify a decorated Schröder path with its underlying Dyck path and we will partly do this by calling the path $P$ and the underlying Dyck path $D$.

Dyck paths may be encoded by the area sequence $\left(a_{1}(D), a_{2}(D), \ldots, a_{n}(D)\right)$ where $a_{i}(D)$ is the number of full cells in the the $i^{t h}$ row which are above the diagonal but below the 
Dyck path. The area sequence of the Dyck paths are characterized by the property that $a_{1}(D)=0$ and $0 \leq a_{i+1}(D) \leq a_{i}(D)+1$ for $1 \leq i<n$. The area statistic on Dyck paths is $\operatorname{area}(D)=\sum_{i=1}^{n} a_{i}(D)$.

The diagonal inversion statistic on Dyck paths is the number of pairs $(i, j)$ with $i<j$ such that either $a_{i}(D)=a_{j}(D)$ or $a_{i}(D)=a_{j}(D)+1$ (the diagonal inversions of the Dyck path). Order the rows from largest area value to smallest and from right to left. The $i^{\text {th }}$ row in this order has area $a_{k}(D)$ for some $k$ and let $b_{i}(D)$ be the number of diagonal inversions of the form $(k, j)$ with $a_{k}(D)=a_{j}(D)$ or $(j, k)$ with $a_{j}(D)=a_{k}(D)+1$. The $b_{i}(D)$ represents the number of diagonal inversions between the $i^{\text {th }}$ vertical step in this order and all those that come before. $\operatorname{Set} \operatorname{dinv}(D)=\sum_{i=1}^{n} b_{i}(D)$.

Example 2. To ensure that that the definitions are clear to this point we list the sequences for the Dyck paths pictured in Figure 3. The $a$-sequence is

$$
(0,1,2,2,1,1,2,0,1,1,2,1,0,1,2),
$$

and the $b$-sequence is

$$
(0,1,2,3,4,4,5,5,6,6,7,6,6,4,2) \text {. }
$$

The $a$ and $b$ sequences of the right Dyck path have a clear relationship to the left Dyck path. The statistics for the right path have the $a$-sequence given by

$$
(0,1,1,2,1,0,1,2,0,1,1,0,0,1)
$$

and the $b$-sequence is

$$
(0,1,2,3,4,4,5,5,6,6,7,6,6,4)
$$

found by deleting the last entry.

The combinatorial interpretation of the symmetric function coefficients are in terms of decorated Schröder paths. The set of o-decorated Schröder paths are Dyck paths where each peak except the rightmost one in the highest diagonal can be replaced with a $N E$ diagonal step and each vertical segment that is not a peak can be labeled with a $\circ$ or not. Denote the set of o-decorated Schröder paths in an $n \times n$ square by $\mathbf{S c h}_{n}^{\circ}$ and the set of Dyck paths of the same size by $\mathcal{D}_{n}$. The cardinality of $\mathbf{S c h}_{n}^{\circ}$ is equal to $\frac{2^{n-1}}{n+1}\left(\begin{array}{c}2 n \\ n\end{array}\right)$. This enumeration follows because for each Dyck path in $\mathcal{D}_{n}$ there are $2^{n-1}$ corresponding Schröder paths since each of the $n-1$ vertical edges of the Dyck path (not the rightmost highest peak) has two choices as being either labeled/diagonal or not labeled/diagonal.

A o-decorated rise on a o-decorated Schröder path is a row where the first vertical segment of two consecutive vertical segments has a o-decoration (i.e. a o-decorated row with $\left.a_{i}(D)=a_{i+1}(D)-1\right)$. Let $\operatorname{Rise}_{\circ}(P)$ be the set of indices of the rows of the odecorated rises and $\operatorname{rise}_{\circ}(P)=\left|\operatorname{Rise}_{\circ}(P)\right|$ be the number of o-decorated rises. We will also set for o-decorated Schröder paths, $\operatorname{area}_{\circ}(P)=\operatorname{area}(D)-\sum_{i \in \operatorname{Rise}_{\circ}(P)} a_{i+1}(D)=\operatorname{area}(D)-$ rise $_{\circ}(D)-\sum_{i \in \operatorname{Rise}_{\circ}(D)} a_{i}(D)$.

There is a reading order of the vertical segments of a Dyck path which are ordered by reading them from the highest diagonal from right to left. We will denote the set of indices 
of the diagonal edges in a corresponding Schröder path $P$ (following the reading order) by $\operatorname{Diag}_{\circ}(P)$ and the number of o-decorated peaks by $\operatorname{diag}_{\circ}(P)=\left|\operatorname{Diag}_{\circ}(P)\right|$.

We note that because we restricted in our o-decorated Schröder paths that the rightmost peak in the highest diagonal cannot be a $N E$-diagonal edge, $1 \notin \operatorname{Diag}_{\circ}(P)$. Also remark that rows that form a peak in a Dyck path will have $b_{i}(D)>b_{i-1}(D)$ (except where $\left.b_{1}(D)=0\right)$. A peak of a Dyck path will have a diagonal inversion with all the same positions that the preceding vertical segment had, plus one with that previous vertical segment.

Denote a restricted diagonal inversion statistic on o-decorated Schröder paths

$$
\operatorname{dinv}_{\circ}(P)=\operatorname{dinv}(D)-\sum_{i \in \operatorname{Diag}_{\circ}(P)} b_{i}(D)
$$

Define the following multivariate analogues of $\frac{2^{n-1}}{n+1}\left(\begin{array}{c}2 n \\ n\end{array}\right)$,

$$
\begin{aligned}
\operatorname{Cat}_{n}^{\prime}(q, t, z, w)= & \sum_{D \in \mathcal{D}_{n}} q^{\operatorname{dinv}(D)} t^{\operatorname{area}(D)} \prod_{b_{i}(D)>b_{i-1}(D)}\left(1+z / q^{b_{i}(D)}\right) \\
& \times \prod_{a_{i}(D)>a_{i-1}(D)}\left(1+w / t^{a_{i}(D)}\right) \\
= & \sum_{P \in \mathbf{S c h}_{n}^{\circ}} q^{\operatorname{dinv}_{\circ}(P)} t^{\operatorname{area}_{\circ}(P)} z^{\operatorname{diag}_{\circ}(P)} w^{\text {riseo }_{\circ}(P)} .
\end{aligned}
$$

There is a compositional refinement that we are focusing on in this paper. For a odecorated Dyck path $D$ with rise $(D)=\ell$, define the rise-touch composition of $D$ to be the sequence of numbers of vertical steps which are not o-decorated rises between the places the path touches the diagonal. This is more simply determined by looking at the usual touch composition for the Dyck path without the decorations and then subtracting from each part of the composition the number of o-decorated rises in each segment. Where it is necessary to abbreviate, the rise-touch composition will be denoted touch $(D)$. It is the case that $\operatorname{touch}(D)$ is a composition of $n-\ell$.

Example 3. To ensure that the combinatorial object and the definitions that we are considering are clear, consider the Schröder path that appears in Figure1. The Schröder path $P$ has an underlying Dyck path $D$ and the $a$-sequence for this path is $(0,1,1,2,0,1,1,0$, $0,1,2,2,3,3,4,2)$ and the $b$-sequence for this path is $(0,0,1,2,1,2,3,1,2,3,3,4,4,5,4,3)$. The $\operatorname{area}(D)=23$ and the so the $\operatorname{area}_{\circ}(P)=23-(1+2+1+3+4)=12$. The $\operatorname{dinv}(D)=38$ and the peaks corresponding to the indices $3,7,9$ in the reading order are $N E$-diagonal edges and so $\operatorname{dinv}_{\circ}(P)=38-(1+3+2)=32$.

We next define a generating function for the o-decorated Dyck paths such that the risetouch composition is equal to $\alpha$. Fix non-negative integers $k$ and $\ell$ and a composition $\alpha$. 
Then set

$$
\operatorname{Cat}_{\alpha, k, \ell}^{\prime}(q, t)=\sum_{\substack{P \in \mathbf{S c h}_{|\alpha|+\ell}^{\circ} \\ \operatorname{diag}_{\circ}(P)=k \\ \operatorname{touch}_{\circ}(P)=\alpha}} q^{\operatorname{dinv}_{\circ}(P)} t^{\text {area }_{\circ}(P)}
$$

By definition,

$$
\operatorname{Cat}_{n}^{\prime}(q, t, z, w)=\sum_{k, \ell: k+\ell \leq n-1} \sum_{\alpha \models n-\ell} \operatorname{Cat}_{\alpha, k, \ell}^{\prime}(q, t) z^{k} w^{\ell} .
$$

this is because if there are $\ell$ rises which are o-decorated, then the rise-touch composition of the o-decorated Dyck path will be some composition $\alpha$ of $n-\ell$ and so both the left and right hand side of the equation is equal to a weighted sum over all o-decorated Dyck paths of size $n$.

Example 4. The following 8 Schröder paths have touch $(P)=(2,2)$. The third path in each row has $\operatorname{area}_{\circ}(P)=3$, the others have $\operatorname{area}_{\circ}(P)=2$.
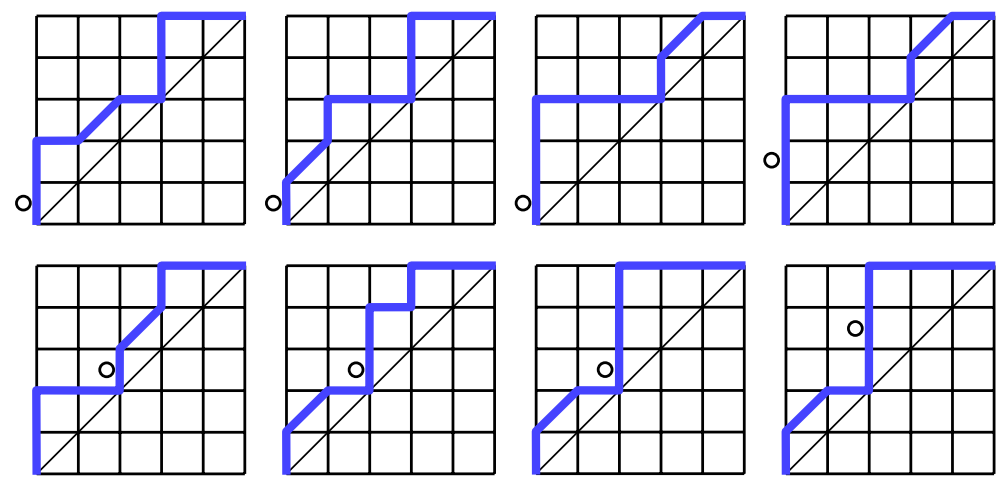

The first two of these paths have $b$-sequence $(0,1,2,2,1)$, the second two have a $b$-sequence $(0,1,1,1,1)$. In the bottom row the first two paths have $b$-sequence $(0,1,2,1,1)$ and the last two have $b$-sequence $(0,0,1,1,1)$. As a consequence the $\operatorname{dinv}_{\circ}(P)$ for the paths are $5,4,3,3$ for the top row and 4,3,2,2 for the bottom row.

The generating function for these objects is

$$
\operatorname{Cat}_{(2,2), 1,1}^{\prime}(q, t)=q^{5} t^{2}+q^{4} t^{2}+q^{3} t^{3}+q^{3} t^{2}+q^{4} t^{2}+q^{3} t^{2}+q^{2} t^{3}+q^{2} t^{2} .
$$

3.1. Combinatorial recurrence on $\operatorname{Cat}_{\beta, k, \ell}^{\prime}(q, t)$. We will show in this subsection that for non-negative integers $k, \ell$ such that $k<|\alpha|$ that $\mathrm{Cat}_{\alpha, k, \ell}^{\prime}(q, t)$ satisfies a recurrence involving the first part of the partition. We consider two cases, one where the first part of the composition $\alpha$ is greater than 1 and a second where $\alpha_{1}=1$.

Proposition 5. For $a \geq 1$, and $k, \ell \geq 0$, and a fixed composition $\beta$ we have the combinatorial recurrence,

$$
\operatorname{Cat}_{(a+1, \beta), k, \ell}^{\prime}(q, t)=t^{a} q^{\ell(\beta)} \sum_{\gamma \models a} \operatorname{Cat}_{(\beta, \gamma), k, \ell}^{\prime}(q, t)+t^{a} q^{\ell(\beta)} \sum_{\gamma \models a+1} \operatorname{Cat}_{(\beta, \gamma), k, \ell-1}^{\prime}(q, t)
$$


Proof. To prove equation (37), we will divide the set of o-decorated Dyck paths into two subsets: either the first vertical step of the o-decorated Dyck path is decorated (Case 1) or it is not decorated (Case 2). On both sets we will perform a cyclic rotation as described at the beginning of this section. We will refer to the piece of the Dyck path up to the first point that it touches the diagonal as first (the part pictured in red in Figure 3) and the piece of the Dyck path after the first part as rest (the part pictured in blue in Figure 3).

The circle decorations travel with the vertical edges of the path and the vertical edge which is deleted may either be decorated or not. The sequence of $b$-values for the cyclically rotated path is exactly the same as the $b$-sequence for the original path except the last entry (which corresponds to the deleted edge) is deleted. This is because diagonal inversions within the first piece or within the rest piece are still diagonal inversions after the cyclic rotation and diagonal inversions between the first piece and the rest piece will switch from being on the same diagonal to being on the diagonal below (and vice versa).

We also remark that the rightmost peak in the highest diagonal in the path before rotation will remain the rightmost peak in the highest diagonal in the path after the operation. Therefore the condition that the rightmost peak in the highest diagonal is not a NE edge is a condition which must hold and come from the base case.

Case 1: Cyclic rotation gives a bijection between o-decorated Schröder paths $P$ with risetouch composition $(a+1, \beta)$ and $\operatorname{diag}_{\circ}(P)=k$ and $\operatorname{rise}_{\circ}(P)=\ell$ where the first vertical step is not decorated and o-decorated Schröder paths $P^{\prime}$ with rise-touch composition of the form $(\beta, \gamma)$ with $\gamma \models a$ and $\operatorname{diag}_{\circ}\left(P^{\prime}\right)=k$ and $\operatorname{diag}_{\circ}\left(P^{\prime}\right)=\ell$.

Say that there are $r$ o-decorated rises in the first piece. Since the first vertical step is not decorated, after deleting the first edge from a piece of a Dyck path which touches the diagonal after $a+r+1$ steps, the rise-touch composition will be a composition $\gamma$ of size $a$ and deleting the first vertical step has the effect of removing $a$ cells contributing to the area (one for each peak or non-decorated rise row in the first piece of the Dyck path), so $\operatorname{area}_{\circ}(D)=\operatorname{area}_{\circ}\left(D^{\prime}\right)+a$. Moreover since there is one diagonal inversion of the form $(1, j)$ for each $j>1$ with $a_{j}(D)=0$, then $b_{n}(D)=\ell(\beta)$ (where $n$ is equal to the length of $D$ ) and $\operatorname{dinv}_{\circ}(D)=\operatorname{dinv}_{\circ}\left(D^{\prime}\right)+\ell(\beta)$.

Example 6. Consider the first part of the Dyck path being the following piece of length 8 . The first part of the rise-touch composition of this Dyck path will be 8 minus the number of rises which are o-decorated in this piece (in this case there are 2).

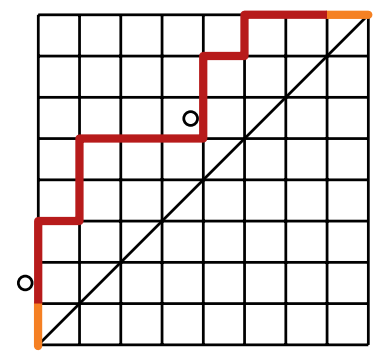


We consider this example to see how the rise-touch composition and o-area is affected by deleting the first vertical step and last horizontal step and ensure that the definitions are clear to this point. Since the o-decorations are in rows 2 and 6 , the o-area contribution from this first piece is 9 and length of the first entry of the rise-touch composition is 6 . Deleting the first vertical and last horizontal step then the rise-touch composition will have contribition $(3,2)$ and the o-area statistic will contribute $4=9-(6-1)$ from this piece.

Case 2: Cyclic rotation also gives a bijection between o-decorated Schröder paths $P$ with rise-touch composition $(a+1, \beta)$ and $\operatorname{diag}_{\circ}(P)=k$ and $\operatorname{rise}_{\circ}(P)=\ell$ where the first vertical step is decorated and o-decorated Dyck paths $P^{\prime}$ with rise-touch composition $(\beta, \gamma)$ with $\gamma \models a+1$ and $\operatorname{diag}_{\circ}\left(P^{\prime}\right)=k$ and $\operatorname{rise}_{\circ}\left(P^{\prime}\right)=\ell-1$. Deleting the o-decoration from the first vertical step will reduce the number of o-decorations by one.

Say that there are $r$ o-decorated rises in the first piece and the Dyck path first touches the diagonal after $a+1+r$ steps. Notice that the rise-touch composition of the first piece of the path after deleting the first step will be a composition $\gamma$ of size $a+1$. Deleting the first vertical step has the effect of removing $a$ cells contributing to the area (one for each peak or non-decorated rise row in the first piece of the Dyck path), so $\operatorname{area}_{\circ}(D)=\operatorname{area}_{\circ}\left(D^{\prime}\right)+a$. Moreover since there is one diagonal inversion of the form $(1, j)$ for each $j>1$ with $a_{j}(D)=0$, then $b_{n}(D)=\ell(\beta)$ and $\operatorname{dinv}_{\circ}(D)=\operatorname{dinv}_{\circ}\left(D^{\prime}\right)+\ell(\beta)$.

Example 7. Consider the same first piece of the Dyck path as in Example 6 but assume now that rows 1,2 and 6 are labelled. The first entry of the rise-touch composition will be 5 which is the same size as the resulting rise-touch composition of $(3,2)$ when we delete the first vertical and last horizontal step.

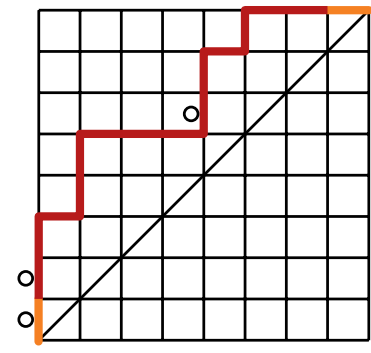

The o-area of the contribution from the first piece is 8 before deleting the first vertical and last horizontal step and it is 4 after.

The two cases are disjoint and together cover all o-decorated Dyck paths and the weights agree between those on the left and right hand side, so equation (37) holds.

Next we consider the case where $\alpha=(1, \beta)$ (that is, the $a=0$ case) and we see that the recurrence is slightly different.

Proposition 8. For $k, \ell \geq 0$, and a composition $\beta$ we have the combinatorial recurrence,

$$
\operatorname{Cat}_{(1, \beta), k, \ell}^{\prime}(q, t)=q^{\ell(\beta)} \operatorname{Cat}_{\beta, k, \ell}^{\prime}(q, t)+\operatorname{Cat}_{\beta, k-1, \ell}^{\prime}(q, t)+q^{\ell(\beta)} \operatorname{Cat}_{(\beta, 1), k, \ell-1}^{\prime}(q, t) .
$$


Proof. In the case that the first part of the rise-touch composition is 1 , there are three types of o-decorated Dyck paths which contribute to this expression: the Dyck path starts with a non-decorated vertical step followed by a horizontal step (Case 1), it begins with a o-decorated vertical step followed by a horizontal step (Case 2) or it begins with some number of o-decorated rises followed by a peak followed by the same number of horizontal steps (Case 3) (in the picture below, the example representing this case has two o-decorated rises, but in general there are potentially between 1 and $\ell$ o-decorated rises and the peak can potentially be a $N E$-diagonal step).

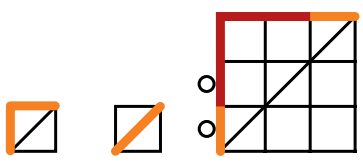

Case 1: The o-decorated Schröder paths $P$ which begin with a non-decorated vertical step followed by a horizontal step with rise-touch composition of the form $(1, \beta)$ and $\operatorname{diag}_{\circ}(P)=$ $k$ and $\operatorname{rise}_{\circ}(P)=\ell$ are in bijection with the o-decorated Schröder paths $P^{\prime}$ with rise-touch composition $\beta$ and $\operatorname{diag}_{\circ}(P)=k$ and $\operatorname{rise}_{\circ}(P)=\ell$ by removing the first vertical and horizontal steps. Since there are $\ell(\beta)$ diagonal inversions of the form $(1, j)$ for each $j>1$ with $a_{j}(D)=0$, then $\operatorname{dinv}_{\circ}(P)=\operatorname{dinv}_{\circ}\left(P^{\prime}\right)+\ell(\beta)$. The area doesn't change by deleting the first vertical and horizontal step so $\operatorname{area}_{\circ}(P)=\operatorname{area}_{\circ}\left(P^{\prime}\right)$.

Case 2: The o-decorated Schröder paths $P$ with rise-touch composition of the form $(1, \beta)$ which begin with a $N E$-diagonal step and $\operatorname{diag}_{\circ}(P)=k$ and $\operatorname{rise}_{\circ}(P)=\ell$ are in bijection with the o-decorated Schröder paths $P^{\prime}$ with rise-touch composition $\beta$, $\operatorname{diag}_{\circ}(P)=k-1$ and $\operatorname{rise}_{\circ}(P)=\ell$. The bijection is simply to remove the first vertical and horizontal steps (and hence one of the decorations on the peaks). Since the first step of the path is a $N E$-diagonal, it does not contribute to the o-dinv statistic and $\operatorname{dinv}_{\circ}(P)=\operatorname{dinv}_{\circ}\left(P^{\prime}\right)$. Moreover the o-area does not change by removing the first vertical and horizontal step so $\operatorname{area}_{\circ}(P)=\operatorname{area}_{\circ}\left(P^{\prime}\right)$.

Case 3: The o-decorated Schröder paths $P$ with rise-touch composition equal to $(1, \beta)$ which begin with a sequence of o-decorated rises, followed by a peak or a $N E$-diagonal step followed by horizontal steps to return to the diagonal with $\operatorname{diag}_{\circ}(P)=k$ and $\operatorname{rise}_{\circ}(P)=\ell$ are in bijection with the Dyck paths $P^{\prime}$ with rise-touch composition $(\beta, 1), \operatorname{diag}_{\circ}(P)=k$ and $\operatorname{rise}_{\circ}(P)=\ell-1$ by a cyclic rotation described at the beginning of this section. We note that the o-area does not change with the cyclic rotation because the first row is a o-decorated rise so $\operatorname{area}_{\circ}(P)=\operatorname{area}_{\circ}\left(P^{\prime}\right)$. Since there is one diagonal inversion of the form $(1, j)$ for each $j>1$ with $a_{j}(D)=0$, then $\operatorname{dinv}_{\circ}(P)=\operatorname{dinv}_{\circ}\left(P^{\prime}\right)+\ell(\beta)$.

The three cases are disjoint, they cover all possible o-decorated Dyck paths with risetouch composition beginning with a 1 and the weights on the left hand side of the equation agree with those on the right hand side, hence equation 28 holds. 


\section{THE SYMMETRIC FUNCTION RECURRENCE}

In this section we will provide a proof of the following symmetric function identity that agrees with the combinatorial recurrence on the generating function for o-decorated Schröder paths.

Theorem 9. For $k \geq 0$, and for integers $d, r, m$,

$$
\begin{gathered}
\mathbb{C}_{k+1}^{*} \Delta_{h_{d}} \nabla\left(e_{r}^{*} h_{m}^{*}\right)=t^{k} \mathbb{B}_{k}^{*} \Delta_{h_{d}} \nabla\left(e_{r}^{*} h_{m-1}^{*}\right)+t^{k} \mathbb{B}_{k+1}^{*} \Delta_{h_{d-1}} \nabla\left(e_{r}^{*} h_{m}^{*}\right) \\
+\chi(k=0) \Delta_{h_{d}} \nabla\left(e_{r-1}^{*} h_{m}^{*}\right) .
\end{gathered}
$$

Notice that at $d=0$, one of the terms is equal to 0 . This case of this identity is equivalent to the recurrence used in GXZ10 to prove the Schröder case of the compositional shuffle conjecture.

We have as a consequence the following expression of coefficients which have combinatorial meaning.

Corollary 10. For non-negative integers $k, \ell$ and $a$ and for a composition $\beta$,

$$
\begin{aligned}
& \left\langle\Delta_{h_{\ell}} \nabla \mathbb{C}_{a+1}\left(C_{\beta}\right), s_{k+1,1^{|\beta|+a-k}}\right\rangle=t^{a} q^{\ell(\beta)} \sum_{\gamma \models a}\left\langle\Delta_{h_{\ell}} \nabla C_{\beta, \gamma}, s_{k+1,1|\beta|+a-k-1}\right\rangle \\
& +t^{a} q^{\ell(\beta)} \sum_{\gamma \models a+1}\left\langle\Delta_{h_{\ell-1}} \nabla C_{\beta, \gamma}, s_{k+1,1|\beta|+a-k}\right\rangle+\chi(a=0)\left\langle\Delta_{h_{\ell}} \nabla\left(C_{\beta}\right), s_{k, 1|\beta|-k}\right\rangle .
\end{aligned}
$$

Proof. This identity is derived by taking the $*$-scalar product with $C_{\beta}$ on both sides of the equation $(29)-(30)$. We begin by taking the $*$-scalar product of $C_{\beta}$ with the left hand side of $(29)$. We note that since $\left\{\tilde{H}_{\mu}\right\}_{\mu}$ is an orthogonal basis with respect to the $*$-scalar product and are eigenvectors of the operators $\nabla$ and $\Delta_{h_{\ell}}$, these operators are self dual with respect to the $*$-scalar product and commute with each other.

$$
\left\langle C_{\beta}, \mathbb{C}_{a+1}^{*} \Delta_{h_{\ell}} \nabla\left(e_{r}^{*} h_{n}^{*}\right)\right\rangle_{*}=\left\langle\Delta_{h_{\ell}} \nabla \mathbb{C}_{a+1}\left(C_{\beta}\right), e_{r}^{*} h_{n}^{*}\right\rangle_{*}=\left\langle\Delta_{h_{\ell}} \nabla \mathbb{C}_{a+1}\left(C_{\beta}\right), h_{r} e_{n}\right\rangle .
$$

Similarly, the scalar product with the other three terms of the right hand side of (29)(30) have expressions involving only $C_{\alpha}$ once equation $(18)$ is applied to the expressions of the form $\mathbb{B}_{m}\left(C_{\beta}\right)$. We conclude that

$$
\begin{aligned}
& \left\langle\Delta_{h_{\ell}} \nabla \mathbb{C}_{a+1}\left(C_{\beta}\right), h_{k+1} e_{|\beta|+a-k}\right\rangle=t^{a} q^{\ell(\beta)} \sum_{\gamma \models a}\left\langle\Delta_{h_{\ell}} \nabla C_{\beta, \gamma}, h_{k+1} e_{|\beta|+a-k-1}\right\rangle \\
& +t^{a} q^{\ell(\beta)} \sum_{\gamma \models a+1}\left\langle\Delta_{h_{\ell-1}} \nabla C_{\beta, \gamma}, h_{k+1} e_{|\beta|+a-k}\right\rangle+\chi(a=0)\left\langle\Delta_{h_{\ell}} \nabla\left(C_{\beta}\right), h_{k} e_{|\beta|-k}\right\rangle .
\end{aligned}
$$

Equations (31)-(32) then follow because $s_{k+1,1^{|\beta|+a-k}}=\sum_{r=0}^{|\beta|+a-k}(-1)^{r} h_{k+1+r} e_{|\beta|+a-k-r}$.

Note that in the case that $k=0$ and $r=1$ that $s_{r-1,1^{n+1-r}}$ is 0 if $n>0$ and $s_{r-1,1^{n+1-r}}=$ 1 if $n=0$. 
Theorem 11. For non-negative integers $k$ and $\ell$ and a composition $\alpha$,

$$
\operatorname{Cat}_{\alpha, k, \ell}^{\prime}(q, t)=\left\langle\Delta_{h_{\ell}} \nabla\left(C_{\alpha}\right), s_{k+1,1^{|\alpha|-k-1}}\right\rangle
$$

Proof. We have just established in the previous section (combining Propositions 5 and 8 ) that

$$
\begin{aligned}
\operatorname{Cat}_{(a+1, \beta), k, \ell}^{\prime}(q, t)= & t^{a} q^{\ell(\beta)} \sum_{\gamma \models a} \operatorname{Cat}_{(\beta, \gamma), k, \ell}^{\prime}(q, t)+t^{a} q^{\ell(\beta)} \sum_{\gamma \models a+1} \operatorname{Cat}_{(\beta, \gamma), k, \ell-1}^{\prime}(q, t) \\
& +\chi(a=0) \operatorname{Cat}_{\beta, k-1, \ell}^{\prime}(q, t) .
\end{aligned}
$$

This combinatorial recurrence agrees with Corollary 10 in the sense that if

$$
\operatorname{Cat}_{(\beta, \gamma), k, \ell}^{\prime}(q, t)=\left\langle\Delta_{h_{\ell}} \nabla C_{\beta, \gamma}, s_{k+1,1|\beta|+a-k-1}\right\rangle
$$

and

$$
\operatorname{Cat}_{(\beta, \gamma), k, \ell-1}^{\prime}(q, t)=\left\langle\Delta_{h_{\ell-1}} \nabla C_{\beta, \gamma}, s_{k+1,1|\beta|+a-k}\right\rangle
$$

and

$$
\operatorname{Cat}_{\beta, k-1, \ell}^{\prime}(q, t)=\left\langle\Delta_{h_{\ell}} \nabla\left(C_{\beta}\right), s_{k, 1|\beta|-k}\right\rangle
$$

then

$$
\operatorname{Cat}_{(a+1, \beta), k, \ell}^{\prime}(q, t)=\left\langle\Delta_{h_{\ell}} \nabla \mathbb{C}_{a+1}\left(C_{\beta}\right), s_{k+1,1|\beta|+a-k}\right\rangle .
$$

The indices of the right hand side of this recurrence have the property that either the value of $\ell$ is lower or the size of the composition $\alpha$ is smaller. Therefore we proceed by induction by assuming that equation (36) holds true for compositions of smaller size and smaller values of $\ell$. Then it remains to show that it is true for a base case.

We note that if $\alpha=(1)$, then

$$
\left\langle\Delta_{h_{\ell}} \nabla\left(C_{1}\right), s_{k+1,1^{-k}}\right\rangle=1
$$

if and only if $k=0$ and it is equal to 0 otherwise. Similarly, $\operatorname{Cat}_{(1), k, \ell}^{\prime}(q, t)=1$ if and only if $k=0$ (and 0 otherwise) because the generating function for o-decorated Schröder paths has one term for the Schröder path consisting of $\ell$ o-decorated rises, a peak, followed by horizontal steps back to the diagonal.

In order to prove our symmetric function identity from Theorem 9 we break the calculation into lemmas that will hopefully make a long calculation a little easier to follow.

Lemma 12. For integers $d$ and partitions $\mu$ and $\nu$,

$$
\frac{T_{\nu}}{w_{\nu}} h_{d}\left[B_{\mu}\right] \tilde{H}_{\mu}\left[D_{\nu}\right]=\sum_{a \geq 0}(-1)^{a} h_{d-a}\left[\frac{1}{M}\right] \sum_{\substack{\gamma \supseteq \nu \\|\gamma|=|\nu|+a}} \tilde{H}_{\mu}\left[D_{\gamma}\right] \frac{T_{\gamma}}{w_{\gamma}} c_{\gamma \nu}^{e_{a}^{\perp}} .
$$


Proof. First we apply equation (2) to $h_{d}\left[B_{\mu}\right]$ (the alphabet addition formula) and show that

$$
h_{d}\left[B_{\mu}\right]=h_{d}\left[\frac{M B_{\mu}-1}{M}+\frac{1}{M}\right]=\sum_{a \geq 0} h_{a}\left[\frac{D_{\mu}}{M}\right] h_{d-a}\left[\frac{1}{M}\right] .
$$

To the left hand side of equation (39) we apply the reciprocity formula (9), and then use the generalized Pieri coefficients that were introduced in GarHag02 from equation (13), and then reapply the reciprocity formula to derive

$$
\begin{aligned}
\frac{T_{\nu}}{w_{\nu}} h_{d}\left[B_{\mu}\right] \tilde{H}_{\mu}\left[D_{\nu}\right] & =(-1)^{|\mu|+|\nu|} \frac{T_{\mu}}{w_{\nu}} \sum_{a \geq 0} h_{a}\left[\frac{D_{\mu}}{M}\right] \tilde{H}_{\nu}\left[D_{\mu}\right] h_{d-a}\left[\frac{1}{M}\right] \\
& =(-1)^{|\mu|+|\nu|} \frac{T_{\mu}}{w_{\nu}} \sum_{a \geq 0} \sum_{\substack{\gamma \supset \nu \\
|\gamma|=|\nu|+a}} d_{\gamma \nu}^{\omega e_{a}^{*}} \tilde{H}_{\gamma}\left[D_{\mu}\right] h_{d-a}\left[\frac{1}{M}\right] \\
& =\frac{T_{\mu}}{w_{\nu}} \sum_{a \geq 0} \sum_{\substack{\gamma \supset \nu \\
|\gamma|=|\nu|+a}}(-1)^{a} \frac{T_{\gamma}}{T_{\mu}} \tilde{H}_{\mu}\left[D_{\gamma}\right] h_{d-a}\left[\frac{1}{M}\right] d_{\gamma \nu}^{\omega e_{a}^{*}} .
\end{aligned}
$$

Now recall that we can convert the $d_{\gamma \nu}^{f}$ coefficients to $c_{\gamma \nu}^{g \perp}$ coefficients using equation (14), the resulting equation is equal to the right hand side of the equation stated in (39).

Now the coefficients $c_{\gamma \nu}^{e_{a} \perp}$ have the sum over $\nu$ that was also calculated by Garsia and Haglund GarHag02] and we need this expression that we state in the following lemma.

Lemma 13. For a a non-negative integer and for a fixed partition $\gamma$,

$$
\sum_{\substack{\nu \subseteq \gamma \\|\nu|=|\gamma|-a}} c_{\gamma \nu}^{e_{a} \perp}=e_{a}\left[B_{\gamma}\right]
$$

Proof. Of course if $|\gamma|<a$ then both the left and right hand sides of this expression are equal to 0 . From the identity in equation (15) in the special case of $g=e_{a}$ we have

$$
\sum_{\substack{\nu \subseteq \gamma \\|\nu|=|\gamma|-a}} c_{\gamma \nu}^{e_{a} \perp}=\left.\nabla^{-1} h_{a}\left[\frac{X-\epsilon}{M}\right]\right|_{X \rightarrow D_{\gamma}} .
$$

We know that $\nabla^{-1} h_{a}\left[\frac{X}{M}\right]=e_{a}\left[\frac{X}{M}\right]$ and hence we apply the alphabet addition formulas to simplify the right hand side of the equation 45 as

$$
\begin{aligned}
\left.\nabla^{-1} h_{a}\left[\frac{X-\epsilon}{M}\right]\right|_{X \rightarrow D_{\gamma}} & =\left.\sum_{b \geq 0} \nabla^{-1} h_{a-b}\left[\frac{X}{M}\right] h_{b}\left[\frac{-\epsilon}{M}\right]\right|_{X \rightarrow D_{\gamma}} \\
& =\sum_{b \geq 0} e_{a-b}\left[\frac{D_{\gamma}}{M}\right] e_{b}\left[\frac{1}{M}\right]=e_{a}\left[\frac{M B_{\gamma}-1}{M}+\frac{1}{M}\right]=e_{a}\left[B_{\gamma}\right] .
\end{aligned}
$$


The following result gives us an expression for a kernel which we can use to apply the $\mathbb{B}$ and $\mathbb{C}$ operators.

Proposition 14. For non-negative integers $d, r$ and $n$

$$
\Delta_{h_{d}} \nabla\left(e_{r}^{*} h_{n}^{*}\right)=\sum_{a \geq 0} \sum_{\gamma}(-1)^{r+a} e_{n+r}\left[\frac{X D_{\gamma}}{M}\right] h_{d-a}\left[\frac{1}{M}\right] h_{n+a-|\gamma|}\left[\frac{-1}{M}\right] \frac{T_{\gamma}}{w_{\gamma}} e_{a}\left[B_{\gamma}\right]
$$

where the sum over $\gamma$ is over all partitions of size smaller than or equal to $n+a$.

Proof. We begin by introducing an extra set of variables $W$ into the expression and using the fact that $e_{r}^{*}[X] h_{n}^{*}[X]=\left\langle e_{n+r}^{*}[X W], h_{r}[W] e_{n}[W]\right\rangle$ where the scalar product is taken with respect to symmetric functions in the variables $W$. Then equation (7) implies

$$
\begin{aligned}
\Delta_{h_{d}} \nabla\left(e_{r}^{*} h_{n}^{*}\right) & =\left\langle\Delta_{h_{d}} \nabla e_{n+r}^{*}[X W], h_{r}[W] e_{n}[W]\right\rangle \\
& =\sum_{\mu \vdash n+r} \frac{h_{d}\left[B_{\mu}\right] T_{\mu} \tilde{H}_{\mu}[X]}{w_{\mu}}\left\langle\tilde{H}_{\mu}[W], h_{r}[W] e_{n}[W]\right\rangle .
\end{aligned}
$$

Now a special case of the Macdonald coefficients that are known (see Mac95] Exercise 2 p. 362) is the scalar product $\left\langle\tilde{H}_{\mu}[W], h_{r}[W] e_{n}[W]\right\rangle=e_{n}\left[B_{\mu}\right]$. To apply our Lemma 12 we need an expression with $D_{\mu}=M B_{\mu}-1$, hence by the alphabet addition formulae, we have

$$
\begin{aligned}
& =\sum_{\mu \vdash n+r} \frac{h_{d}\left[B_{\mu}\right] T_{\mu} \tilde{H}_{\mu}[X]}{w_{\mu}} e_{n}\left[B_{\mu}\right] \\
& =\sum_{\mu \vdash n+r} \frac{h_{d}\left[B_{\mu}\right] T_{\mu} \tilde{H}_{\mu}[X]}{w_{\mu}} e_{n}^{*}\left[\left(M B_{\mu}-1\right)+1\right] \\
& =\sum_{\mu \vdash n+r} \sum_{k \geq 0} \frac{h_{d}\left[B_{\mu}\right] T_{\mu} \tilde{H}_{\mu}[X]}{w_{\mu}} e_{k}^{*}\left[D_{\mu}\right] e_{n-k}^{*}[1] .
\end{aligned}
$$

We can then expand the expression $e_{k}^{*}\left[D_{\mu}\right]=\sum_{\nu \vdash k} \frac{\tilde{H}_{\nu}\left[D_{\mu}\right]}{w_{\nu}}$ so that we can apply the reciprocity formula from equation (9).

$$
\begin{aligned}
& =\sum_{\mu \vdash n+r} \sum_{k \geq 0} \sum_{\nu \vdash k} \frac{h_{d}\left[B_{\mu}\right] T_{\mu} \tilde{H}_{\mu}[X]}{w_{\mu}} \frac{\tilde{H}_{\nu}\left[D_{\mu}\right]}{w_{\nu}} e_{n-k}^{*}[1] \\
& =\sum_{\mu \vdash n+r} \sum_{k \geq 0} \sum_{\nu \vdash k}(-1)^{n+r+k} \frac{h_{d}\left[B_{\mu}\right] \tilde{H}_{\mu}[X]}{w_{\mu}} \frac{T_{\nu} \tilde{H}_{\mu}\left[D_{\nu}\right]}{w_{\nu}} e_{n-k}^{*}[1] .
\end{aligned}
$$

At this point we can apply equation $(39)$ and simplify the power of -1 by the expression $(-1)^{n+k} e_{n-k}^{*}[1]=h_{n-k}^{*}[-1]$. We also combine the sum over $k \geq 0$ and $\nu \vdash k$ to just be a sum over all partitions $\nu$. The sum is actually finite because the expression $h_{n-k}^{*}[-1]$ is 
equal to 0 if $|\nu|>n$. We then interchange the sum over $\nu$ and $\gamma$ then we have the following manipulation of equation (54) to arrive at the expression stated in the theorem.

$$
\begin{aligned}
& =\sum_{\mu \vdash n+r} \sum_{\nu}(-1)^{r} \frac{\tilde{H}_{\mu}[X]}{w_{\mu}} h_{n-|\nu|}^{*}[-1] \sum_{a \geq 0}(-1)^{a} h_{d-a}^{*}[1] \sum_{\substack{\gamma \supset \nu \\
|\gamma|=|\nu|+a}} \tilde{H}_{\mu}\left[D_{\gamma}\right] \frac{T_{\gamma}}{w_{\gamma}} c_{\gamma \nu}^{e_{a} \perp} \\
& =\sum_{\mu \vdash n+r} \sum_{\gamma} \sum_{a \geq 0}(-1)^{r+a} \frac{\tilde{H}_{\mu}[X] \tilde{H}_{\mu}\left[D_{\gamma}\right]}{w_{\mu}} h_{n+a-|\gamma|}^{*}[-1] h_{d-a}^{*}[1] \frac{T_{\gamma}}{w_{\gamma}} \sum_{\substack{\nu \subseteq \gamma \\
|\nu|=|\gamma|-a}} c_{\gamma \nu}^{e_{a} \perp} \\
& =\sum_{\gamma} \sum_{a \geq 0}(-1)^{r+a} e_{n+r}^{*}\left[X D_{\gamma}\right] h_{n+a-|\gamma|}^{*}[-1] h_{d-a}^{*}[1] \frac{T_{\gamma}}{w_{\gamma}} e_{a}\left[B_{\gamma}\right] .
\end{aligned}
$$

Now we are able to apply the $\mathbb{C}$ and $\mathbb{B}$ operators to prove Theorem 9 ,

To simplify our calculation, it will help to have expressions for the action of $\mathbb{C}_{m}^{*}$ on $e_{c}\left[\frac{X D_{\gamma}}{M}\right]$.

Lemma 15. For a non-negative integer $c$ and integer $m$,

$$
\mathbb{C}_{m}^{*} e_{c}\left[\frac{X D_{\gamma}}{M}\right]=\sum_{b \geq 0} q^{-b+1}(-1)^{m+1} e_{c-b}\left[\frac{X D_{\gamma}}{M}\right] h_{b}\left[D_{\gamma}\right] e_{b-m}\left[\frac{X}{1-t}\right]
$$

Proof.

$$
\begin{aligned}
\mathbb{C}_{m}^{*} e_{c}\left[\frac{X D_{\gamma}}{M}\right] & =\left.(-q) e_{c}\left[\left(X-\frac{M}{q u}\right) \frac{D_{\gamma}}{M}\right] \Omega\left[\frac{-u X}{1-t}\right]\right|_{u^{-m}} \\
& =\left.\sum_{a \geq 0} \sum_{b \geq 0} u^{a-b} q^{-b+1}(-1)^{a+b+1} e_{c-b}\left[\frac{X D_{\gamma}}{M}\right] h_{b}\left[D_{\gamma}\right] e_{a}\left[\frac{X}{1-t}\right]\right|_{u^{-m}}
\end{aligned}
$$

so when we take the coefficient of $u^{-m}$ in $u^{a-b}$, then $-m=a-b$ or $a=b-m$ and we obtain the expression stated in equation 57.

We also develop a full expression for $\mathbb{B}_{m}^{*}$ on the kernel from Proposition 14 because to prove the theorem we will expand the left hand side of 29 ) and need to recognize when we have the right hand side of that equation.

Lemma 16. For all integers $m, r$ and $a$ and for non negative integer $d$,

$$
\begin{aligned}
& \mathbb{B}_{m}^{*} \Delta_{h_{d}} \nabla\left(e_{r}^{*} h_{n}^{*}\right)=\sum_{a \geq 0} \sum_{b \geq 0} \sum_{\tau}(-1)^{r+a+b+m} e_{b}\left[D_{\tau}\right] e_{n+r-b}\left[\frac{X D_{\tau}}{M}\right] \times \\
& \times e_{b-m}\left[\frac{X}{1-t}\right] h_{d-a}^{*}[1] h_{n+a-|\tau|}^{*}[-1] \frac{T_{\tau}}{w_{\tau}} e_{a}\left[B_{\tau}\right] .
\end{aligned}
$$


Proof. As we did in the previous lemma, we will first develop an expression for the action of $\mathbb{B}_{m}^{*}$ on $e_{n+r}^{*}\left[X D_{\gamma}\right]$.

$$
\begin{aligned}
\mathbb{B}_{m}^{*} e_{n+r}^{*}\left[X D_{\tau}\right] & =\left.e_{n+r}\left[\left(X+\frac{M}{u}\right) \frac{D_{\tau}}{M}\right] \Omega\left[\frac{-u X}{1-t}\right]\right|_{u^{-m}} \\
& =\left.\sum_{a \geq 0} \sum_{b \geq 0} u^{a-b} e_{b}\left[D_{\tau}\right] e_{n+r-b}\left[\frac{X D_{\tau}}{M}\right] h_{a}\left[\frac{-X}{1-t}\right]\right|_{u^{-m}} .
\end{aligned}
$$

Now when we take the coefficient of $u^{-m}$ in this expression $-m=a-b$, hence $a=b-m$ and our expression becomes

$$
\mathbb{B}_{m}^{*} e_{n+r}^{*}\left[X D_{\tau}\right]=\sum_{b \geq 0}(-1)^{b+m} e_{b}\left[D_{\tau}\right] e_{n+r-b}\left[\frac{X D_{\tau}}{M}\right] e_{b-m}\left[\frac{X}{1-t}\right]
$$

Now we apply that expression to the kernel that we derived in Proposition 14 .

$$
\begin{aligned}
& \mathbb{B}_{m}^{*} \Delta_{h_{d}} \nabla\left(e_{r}^{*} h_{n}^{*}\right) \\
&=\sum_{a \geq 0} \sum_{\tau}(-1)^{r+a} \mathbb{B}_{m}^{*} e_{n+r}\left[\frac{X D_{\tau}}{M}\right] h_{d-a}^{*}[1] h_{n+a-|\tau|}^{*}[-1] \frac{T_{\tau}}{w_{\tau}} e_{a}\left[B_{\tau}\right] \\
&=\sum_{a \geq 0} \sum_{b \geq 0} \sum_{\tau}(-1)^{r+a+b+m} e_{n+r-b}\left[\frac{X D_{\tau}}{M}\right] e_{b}\left[D_{\tau}\right] \times \\
& \times e_{b-m}\left[\frac{X}{1-t}\right] h_{d-a}^{*}[1] h_{n+a-|\tau|}^{*}[-1] \frac{T_{\tau}}{w_{\tau}} e_{a}\left[B_{\tau}\right] .
\end{aligned}
$$

We are now prepared to prove Theorem 9 by a direct calculation using the results we have derived above.

Proof. (of Theorem 9) We begin by applying the operator $\mathbb{C}_{k+1}$ to the expression in Proposition 14. Equation (57) with $m \rightarrow k+1, c \rightarrow n+r$ says that it is equal to

$$
\begin{aligned}
& \mathbb{C}_{k+1}^{*} \Delta_{h_{d}} \nabla\left(e_{r}^{*} h_{n}^{*}\right)=\sum_{a \geq 0} \sum_{\gamma} \sum_{b \geq 0} q^{-b+1}(-1)^{r+a+k} e_{n+r-b}^{*}\left[X D_{\gamma}\right] h_{b}\left[D_{\gamma}\right] \times \\
& \times e_{b-k-1}\left[\frac{X}{1-t}\right] h_{d-a}^{*}[1] h_{n+a-|\gamma|}^{*}[-1] \frac{T_{\gamma}}{w_{\gamma}} e_{a}\left[B_{\gamma}\right] .
\end{aligned}
$$

Now we know that since $k \geq 0$, then $b \geq 1$ since $e_{b-k-1}=0$ for $b=0$. In fact, it may be helpful to make the replacement $b \rightarrow b+1$ in $(69)$ and we can apply equation $(12)$ to 
$h_{b+1}\left[D_{\gamma}\right]$.

$$
\begin{aligned}
&=\sum_{a \geq 0} \sum_{\gamma} \sum_{b \geq 0} q^{-b}(-1)^{r+a+k} e_{n+r-b-1}^{*}\left[X D_{\gamma}\right] h_{b+1}\left[D_{\gamma}\right] \times \\
& \times e_{b-k}\left[\frac{X}{1-t}\right] h_{d-a}^{*}[1] h_{n+a-|\gamma|}^{*}[-1] \frac{T_{\gamma}}{w_{\gamma}} e_{a}\left[B_{\gamma}\right] \\
&=\sum_{a \geq 0} \sum_{\gamma} \sum_{b \geq 0} \sum_{\tau \rightarrow \gamma}(-1)^{r+a+k} M t^{b} c_{\gamma \tau}\left(\frac{T_{\gamma}}{T_{\tau}}\right)^{b} e_{n+r-b-1}^{*}\left[X D_{\gamma}\right] \times \\
& \times e_{b-k}\left[\frac{X}{1-t}\right] h_{d-a}^{*}[1] h_{n+a-|\gamma|}^{*}[-1] \frac{T_{\gamma}}{w_{\gamma}} e_{a}\left[B_{\gamma}\right] \\
&-\chi(k=0) \sum_{a \geq 0} \sum_{\gamma}(-1)^{r+a} e_{n+r-1}^{*}\left[X D_{\gamma}\right] h_{d-a}^{*}[1] h_{n+a-|\gamma|}^{*}[-1] \frac{T_{\gamma}}{w_{\gamma}} e_{a}\left[B_{\gamma}\right] .
\end{aligned}
$$

Notice already that equation (74) is equal to $+\chi(k=0) \Delta_{h_{d}} \nabla\left(e_{r-1}^{*} h_{n}^{*}\right)$. Then it remains to expand equations $72-(73)$. For this we use $B_{\gamma}=B_{\tau}+\frac{T_{\gamma}}{T_{\tau}}$ and $D_{\gamma}=D_{\tau}+M \frac{T_{\gamma}}{T_{\tau}}$. In this case $e_{a}\left[B_{\gamma}\right]=e_{a}\left[B_{\tau}\right]+e_{a-1}\left[B_{\tau}\right] \frac{T_{\gamma}}{T_{\tau}}$ and $e_{n}^{*}\left[X D_{\gamma}\right]=\sum_{c \geq 0} e_{n-c}^{*}\left[X D_{\tau}\right] e_{c}\left[X \frac{T_{\gamma}}{T_{\tau}}\right]$. We will also use the identity $t^{b} e_{b-k}\left[\frac{X}{1-t}\right]=t^{k} e_{b-k}\left[\frac{t X}{1-t}\right]$ to show that $72-73$ is equivalent to the following expression:

$$
\begin{gathered}
=t^{k} \sum_{a \geq 0} \sum_{\gamma} \sum_{b \geq 0} \sum_{\tau \rightarrow \gamma} \sum_{c \geq 0}(-1)^{r+a+k} M c_{\gamma \tau}\left(\frac{T_{\gamma}}{T_{\tau}}\right)^{b} e_{n+r-b-1-c}^{*}\left[X D_{\tau}\right] e_{c}\left[X \frac{T_{\gamma}}{T_{\tau}}\right] \times \\
\times e_{b-k}\left[\frac{t X}{1-t}\right] h_{d-a}^{*}[1] h_{n+a-|\gamma|}^{*}[-1] \frac{T_{\gamma}}{w_{\gamma}}\left(e_{a}\left[B_{\tau}\right]+e_{a-1}\left[B_{\tau}\right] \frac{T_{\gamma}}{T_{\tau}}\right) .
\end{gathered}
$$

We will regroup the $T_{\gamma} / T_{\tau}$ terms and break the expression into two separate sums. We will also replace $c_{\gamma \tau}$ with $d_{\gamma \tau}$ using equation (10). Then equations (75)-(76) are equivalent to

$$
\begin{aligned}
&=t^{k} \sum_{a \geq 0} \sum_{\gamma} \sum_{b \geq 0} \sum_{\tau \rightarrow \gamma} \sum_{c \geq 0}(-1)^{r+a+k} d_{\gamma \tau}\left(\frac{T_{\gamma}}{T_{\tau}}\right)^{b+c+1} e_{n+r-b-1-c}^{*}\left[X D_{\tau}\right] e_{c}[X] \times \\
& \times e_{b-k}\left[\frac{t X}{1-t}\right] h_{d-a}^{*}[1] h_{n+a-|\gamma|}^{*}[-1] \frac{T_{\gamma}}{w_{\tau}} e_{a}\left[B_{\tau}\right] . \\
&+t^{k} \sum_{a \geq 0} \sum_{\gamma} \sum_{b \geq 0} \sum_{\tau \rightarrow \gamma} \sum_{c \geq 0}(-1)^{r+a+k} d_{\gamma \tau}\left(\frac{T_{\gamma}}{T_{\tau}}\right)^{b+c+2} e_{n+r-b-1-c}^{*}\left[X D_{\tau}\right] e_{c}[X] \times \\
& \quad \times e_{b-k}\left[\frac{t X}{1-t}\right] h_{d-a}^{*}[1] h_{n+a-|\gamma|}^{*}[-1] \frac{T_{\tau}}{w_{\tau}} e_{a-1}\left[B_{\tau}\right] .
\end{aligned}
$$

In both of these sums, we can interchange the sum over partitions $\gamma$ and then over $\tau \rightarrow \gamma$ to a sum over partitions $\tau$ and then over $\gamma \leftarrow \tau$. In this case the sums of the form $\sum_{\gamma \leftarrow \tau} d_{\gamma \tau}\left(\frac{T_{\gamma}}{T_{\tau}}\right)^{n}=(-1)^{n-1} e_{n-1}\left[D_{\tau}\right]$ (where $n \in\{b+c+1, b+c+2\}$ ) by equation (11) 
since in both of the expressions we have $n>1$ so there is no $\chi(n=0)$ term. Equations 777) -80 are equivalent to

$$
\begin{aligned}
=t^{k} \sum_{a \geq 0} & \sum_{b \geq 0} \sum_{c \geq 0} \sum_{\tau}(-1)^{r+a+k+b+c} e_{b+c}\left[D_{\tau}\right] e_{n+r-b-1-c}^{*}\left[X D_{\tau}\right] e_{c}[X] \times \\
& \times e_{b-k}\left[\frac{t X}{1-t}\right] h_{d-a}^{*}[1] h_{n+a-|\tau|-1}^{*}[-1] \frac{T_{\tau}}{w_{\tau}} e_{a}\left[B_{\tau}\right] . \\
+t^{k} & \sum_{a \geq 0} \sum_{b \geq 0} \sum_{c \geq 0} \sum_{\tau}(-1)^{r+a+k+b+c+1} e_{b+c+1}\left[D_{\tau}\right] e_{n+r-b-1-c}^{*}\left[X D_{\tau}\right] e_{c}[X] \times \\
& \times e_{b-k}\left[\frac{t X}{1-t}\right] h_{d-a}^{*}[1] h_{n+a-|\tau|-1}^{*}[-1] \frac{T_{\tau}}{w_{\tau}} e_{a-1}\left[B_{\tau}\right] .
\end{aligned}
$$

Instead of summing over $b \geq 0$ and $c \geq 0$, we will let $v=b+c$ and sum over $v \geq 0$ and $0 \leq b \leq v$ and let $c=v-b$. These replacements make equations (81)-84) equivalent to

$$
\begin{aligned}
&=t^{k} \sum_{a \geq 0} \sum_{v \geq 0} \sum_{b=0}^{v} \sum_{\tau}(-1)^{r+a+k+v} e_{v}\left[D_{\tau}\right] e_{n+r-v-1}^{*}\left[X D_{\tau}\right] e_{v-b}[X] \times \\
& \times e_{b-k}\left[\frac{t X}{1-t}\right] h_{d-a}^{*}[1] h_{n+a-|\tau|-1}^{*}[-1] \frac{T_{\tau}}{w_{\tau}} e_{a}\left[B_{\tau}\right] . \\
&+t^{k} \sum_{a \geq 0} \sum_{v \geq 0} \sum_{b=0}^{v} \sum_{\tau}(-1)^{r+a+k+v+1} e_{v+1}\left[D_{\tau}\right] e_{n+r-v-1}^{*}\left[X D_{\tau}\right] e_{v-b}[X] \times \\
& \times e_{b-k}\left[\frac{t X}{1-t}\right] h_{d-a}^{*}[1] h_{n+a-|\tau|-1}^{*}[-1] \frac{T_{\tau}}{w_{\tau}} e_{a-1}\left[B_{\tau}\right] .
\end{aligned}
$$

Notice now that the sub expression

$$
\sum_{b=0}^{v} e_{v-b}[X] e_{b-k}\left[\frac{t X}{1-t}\right]=e_{v-k}\left[X+\frac{t X}{1-t}\right]=e_{v-k}\left[\frac{X}{1-t}\right] .
$$

By replacing this in the sum, equations $(85)-(88)$ are equivalent to

$$
\begin{aligned}
&=t^{k} \sum_{a \geq 0} \sum_{v \geq 0} \sum_{\tau}(-1)^{r+a+k+v} e_{v}\left[D_{\tau}\right] e_{n+r-v-1}^{*}\left[X D_{\tau}\right] \times \\
& \times e_{v-k}\left[\frac{X}{1-t}\right] h_{d-a}^{*}[1] h_{n+a-|\tau|-1}^{*}[-1] \frac{T_{\tau}}{w_{\tau}} e_{a}\left[B_{\tau}\right] . \\
&+t^{k} \sum_{a \geq 0} \sum_{v \geq 0} \sum_{\tau}(-1)^{r+a+k+v+1} e_{v+1}\left[D_{\tau}\right] e_{n+r-v-1}^{*}\left[X D_{\tau}\right] \times \\
& \quad \times e_{v-k}\left[\frac{X}{1-t}\right] h_{d-a}^{*}[1] h_{n+a-|\tau|-1}^{*}[-1] \frac{T_{\tau}}{w_{\tau}} e_{a-1}\left[B_{\tau}\right] .
\end{aligned}
$$

We then note that equation $90-91)$ is the right hand side of the expression derived in Lemma 16 with $m \rightarrow k$ and $n \rightarrow n-1$ and hence is equal to $t^{k} \mathbb{B}_{k}^{*} \Delta_{h_{d}} \nabla\left(e_{r}^{*} h_{n-1}^{*}\right)$. As well 
we have that (92)-(93) with $v \rightarrow v-1$ and $a \rightarrow a+1$ is equivalent to the expression

$$
\begin{aligned}
t^{k} \sum_{a \geq-1} \sum_{v \geq 1} \sum_{\tau}(-1)^{r+a+k+v+1} e_{v}\left[D_{\tau}\right] e_{n+r-v}^{*}\left[X D_{\tau}\right] \times \\
\quad \times e_{v-k-1}\left[\frac{X}{1-t}\right] h_{d-a-1}^{*}[1] h_{n+a-|\tau|}^{*}[-1] \frac{T_{\tau}}{w_{\tau}} e_{a}\left[B_{\tau}\right]
\end{aligned}
$$

and with a few exceptions of terms which are equal to 0 (i.e. $a=-1$ and $v=0$ ) this is precisely the right hand side of the expression in Lemma 16 with $m \rightarrow k+1, d \rightarrow d-1$ and hence is equal to $t^{k} \mathbb{B}_{k+1}^{*} \Delta_{h_{d-1}} \nabla\left(e_{r}^{*} h_{n}^{*}\right)$.

\section{REMARKS}

Section 7 of [HRW15] has two conjectures that we do not resolve here. These techniques may potentially be adapted to prove them as well, but some additional work remains.

The first conjecture which does not follow directly from Theorem 11 is a symmetry property that is part of Conjecture 7.1 in [HRW15].

Conjecture 17. For non-negative integers $k, \ell$ and $n>k+\ell$,

$$
\left\langle\Delta_{h_{\ell}} \nabla e_{n-\ell}, s_{k+1,1^{n-\ell-k-1}}\right\rangle=\left\langle\Delta_{h_{k}} \nabla e_{n-k}, s_{\ell+1,1^{n-\ell-k-1}}\right\rangle .
$$

A proof of this result was announced recently by Michele D'Adderio and Anna Vanden Wyngaerd AW17.

A second conjecture is a combinatorial interpretation in terms of a second type of decorated Dyck paths where vertical edges except for the bottom most left one can be decorated. This second combinatorial interpretation does not seem to be compatible with the compositional refinement as it is currently formulated. However it is conjectured that it is compatible with coefficients of the form $\left\langle\Delta_{h_{k}} \nabla E_{n-k, p}, s_{\ell+1,1^{n-k-\ell-1}}\right\rangle$ (see Conjecture 7.2 of [HRW15]) where

$$
E_{n-k, r}=\sum_{\substack{\alpha=n-k \\ \ell(\alpha)=r}} C_{\alpha} .
$$

Potentially recurrences on these coefficients that are compatible with the interpretation with this other type of decorated Dyck path can be derived from Corollary 10 .

The Delta Conjecture is a combinatorial interpretation for the coefficients $\left\langle\Delta_{e_{\ell}} e_{n}, h_{\lambda}\right\rangle$ for $\lambda \vdash n$. We would guess that a different approach than was developed for the Shuffle Conjecture may be needed because the coefficients $\left\langle\Delta_{e_{\ell}} C_{\alpha}, h_{\lambda}\right\rangle$ are not even polynomials in $q$ and $t$ unless $\ell=|\alpha|$ (and this case is the Compositional Shuffle Conjecture).

It seems possible that one of the combinatorial interpretations for the $q, t, w, z$-Catalan might extend to give an interpretation for $\left\langle\Delta_{h_{\ell}} \nabla C_{\alpha}, h_{\lambda}\right\rangle$. In this case, the techniques developed by Carlsson and Mellit CM15] might prove to be useful since those coefficients are compatible with the compositional refinement. The extensions ideally are leading us in the direction of explaining the following conjecture. 
Conjecture 18. For non-negative integers $\ell$ and $n>k+\ell$ and a composition $\alpha=n-\ell$ and a partition $\lambda \vdash n-\ell$,

$$
\left\langle\Delta_{s_{\mu}} \nabla C_{\alpha}, s_{\lambda}\right\rangle
$$

are polynomials in $q$ and $t$ with non-negative integer coefficients.

While there has been significant recent progress on the monomial expansions of $\nabla(f)$ for various symmetric functions $f$, passing to the Schur expansions is an important major hurdle.

\section{REFERENCES}

[AW17] M. D'Adderio, A. Vanden Wyngaerd Decorated Dyck paths, the Delta conjecture, and a new q, tsquare, arXiv:1709.08736

[BG99] F. Bergeron and A. M. Garsia, Science fiction and Macdonald's polynomials, Algebraic methods and $q$-special functions, (Montréal, QC, 1996), CRM Proc. Lecture Notes, vol. 22, Amer. Math. Soc., Providence, RI, 1999, pp. 1-52.

[BGHT99] F. Bergeron, A. M. Garsia, M. Haiman, and G. Tesler, Identities and positivity conjectures for some remarkable operators in the theory of symmetric functions, Methods Appl. Anal. 6 (1999), $363-420$.

[BGLX16] F. Bergeron, A. Garsia, E. Leven, G. Xin, Compositional (km,kn)-Shuffle Conjectures, Inter. Math. Res. Not., Vol. 2016, 42294270 doi:10.1093/imrn/rnv272

[CL06] M. Can, N. Loehr, A proof of the q,t-square conjecture, J. of Comb. Th., Series A Volume 113, Issue 7, October 2006, Pages 1419-1434.

[CM15] E. Carlsson and A. Mellit. A proof of the shuffle conjecture, J. Amer. Math. Soc., to appear, https://doi.org/10.1090/jams/893.

[DGZ13] A. Duane, A. M. Garsia, M. Zabrocki, A new "dinv" arising from the two part case of the Shuffle Conjecture, Journal of Alg. Comb., Volume 37, Issue 4 (2013), Page 683-715. (DOI: 10.1007/s10801012-0382-0)

[EHKK03] E. Egge, J. Haglund, D. Kremer and K. Killpatrick, A Schröder generalization of Haglund's statistic on Catalan paths. Electron. J. of Combin., 10 (2003), Research Paper 16, 21 pages (electronic)

[G92] A. M. Garsia, Orthogonality of Milne's polynomials and raising operators, Discrete Math. 99, Issue 1-3 (1992), 247-264.

[GarHag02] A. M. Garsia and J. Haglund, A proof of the q, t-Catalan positivity conjecture, Discrete Math. 256 (2002), 677-717.

[GarHai93] A. Garsia and M. Haiman, A graded representation model for Macdonald's polynomials Proc. Nat. Acad. Sci. U.S.A. 90 (1993), no. 8, 3607-3610.

[GarHai95] A. Garsia and M. Haiman, A random q, t-hook walk and a sum of Pieri coefficients, J. Combin. Theory, Ser. A 82 (1998), 74-111.

[GarHai96] A. Garsia and M. Haiman, A remarkable q,t-Catalan sequence and q-Lagrange inversion, J. Algebraic Combinatorics 5, (1996), 191-244.

[GHT99] A. M. Garsia, M. Haiman, and G. Tesler, Explicit plethystic formulas for Macdonald q, t-Kostka coefficients, Sém. Lothar. Combin. 42 (1999), Art. B42m, 45 pp. (electronic), The Andrews Festschrift (Maratea, 1998).

[GHXZ16] A. M. Garsia, J. Haglund, G. Xin, M. Zabrocki, Some new applications of the Stanley-Macdonald Pieri Rules, for Stanley@70 (MIT, June 23-27, 2014).

[GXZ10] A. Garsia, G. Xin, and M. Zabrocki, Hall-Littlewood operators in the theory of parking functions and diagonal harmonics, Int. Math. Res. Notices, 2012 (6): 1264-1299. doi: 10.1093/imrn/rnr060

[GXZ14a] A. Garsia, G. Xin, and M. Zabrocki, A three shuffle case of the compositional parking function conjecture, Journal of Combinatorial Theory, Series A 123/1 (2014), pp. 202-238. 
[GXZ14b] A. Garsia, G. Xin, and M. Zabrocki, Proof of the 2-part Compositional Shuffle Conjecture, Progress in Mathematics (Book 257), Birkhäuser; 2014, 538 pages, pp. 227-257.

[Hag03] J. Haglund, Conjectured statistics for the q,t-Catalan numbers, Adv. Math. 175 (2003), no. 2, 319-334.

[Hag04] J. Haglund, A proof of the q,t-Schröder conjecture, Internat. Math. Res. Notices 11 (2004), 525560.

[Hag08] J. Haglund, The q,t-Catalan numbers and the space of diagonal harmonics, University Lecture Series, vol. 41, American Mathematical Society, Providence, RI, 2008, With an appendix on the combinatorics of Macdonald polynomials.

[HMZ12] J. Haglund, J. Morse, M. Zabrocki, A compositional shuffle conjecture specifying touch points of the Dyck path, (arXiv:1008.0828v2), Canad. J. of Math. 64(2012), no. 4, 822-844.

[Hai94] M. Haiman, Conjectures On The Quotient Ring By Diagonal Invariants, J. Algebraic Combin, Volume 3, Issue 1 (1994), 17-76.

[Hai02] M. Haiman, Vanishing theorems and character formulas for the Hilbert scheme of points in the plane, Invent. Math. 149 (2002), 371-407.

[HHLRU05] J. Haglund, M. Haiman, N. Loehr, J. B. Remmel, and A. Ulyanov, A combinatorial formula for the character of the diagonal coinvariants, Duke J. Math. 126 (2005), 195-232.

[Hic10] A. Hicks, Two parking function bijections refining the q, t-Catalan and Schröder recursions, Int. Math. Res. Notices, Volume 2012, No 16, July 2011.

[Hic14] A. Hicks, A parking function bijection supporting the Haglund-Morse-Zabrocki conjectures, Int. Math. Res. Notices, Volume 2014, No 7.

[HRW15] J. Haglund, J. Remmel, A. Wilson, The Delta Conjecture, Trans. Amer. Math. Soc., arXiv:1509.07058v3 .

[LW07] N. A. Loehr and G. Warrington, Square q,t-Lattice Paths and $\nabla\left(p_{n}\right)$, Trans. of the Amer. Math. Soc., Vol. 359, No. 2 (Feb., 2007), pp. 649-669.

[LW08] N. A. Loehr and G. Warrington, Nested quantum Dyck paths and $\nabla\left(s_{\lambda}\right)$, Inter. Math. Res. Not. 2008; Vol 2008: article ID rnm157

[Mac88] I. G. Macdonald, A New Class Of Symmetric Functions, Publ. I.R.M.A. Strasbourg, 372/S20, Actes 20 Séminaire Lotharingien, 1988, 131-171.

[Mac95] I. G. Macdonald, Symmetric functions and Hall polynomials, Oxford Mathematical Monographs, second ed., Oxford Science Publications, The Clarendon Press Oxford University Press, New York, 1995.

[Mel16] A. Mellit, Toric braids and $(m, n)$-parking functions, arXiv:1604.07456 